\title{
Novel Very Large Zenith Angle Observation Technique Performed by the MAGIC Telescopes
}

\author{
levgen Vovk \\ Max-Planck-Institut für Physik, Munich, Germany \\ E-mail: ie.vovkempp.mpg.de
}

\section{Martin Will*}

Max-Planck-Institut für Physik, Munich, Germany

E-mail: mwillampp.mpg.de

\section{Razmik Mirzoyan}

Max-Planck-Institut für Physik, Munich, Germany

E-mail: razmik.mirzoyan@mpp.mpg.de

\section{J. Besenrieder, M. Peresano, P. Temnikov, J. van Scherpenberg, D. Zarić for the MAGIC Collaboration ${ }^{\dagger}$}

We report on the significantly increased observation limits of Imaging Atmospheric Cherenkov Telescopes (IACTs), used for ground-based gamma-ray astronomy in the Very High Energy (VHE) range ( $50 \mathrm{GeV}$ to $50 \mathrm{TeV}$ ). IACTs typically perform air shower observations until zenith angles of about $60^{\circ}$. Beyond that, the column density of air in the atmosphere rapidly increases, and the Cherenkov light absorption starts playing a major role. The absence of a proper calibration method for light transmission restrained researchers from performing regular measurements under very large zenith angles.

MAGIC extends the observation of air showers in Cherenkov light to almost the horizon. To properly calibrate the accumulated data set, we have developed an auxiliary procedure of atmospheric transmission monitoring. This employs optical images of the stellar field next to the source position and provides a good measure of the atmospheric transparency.

Along with longer observation times for a given source, Very Large Zenith Angle (VLZA) technique allows to increase the collection area of IACTs in the TeV energy range of the spectrum of cosmic sources. This is especially important at $\sim 100 \mathrm{TeV}$, where the expected count rates from astrophysical sources are very low. Here we demonstrate that MAGIC Very Large Zenith Angle observations yield a collection area in excess of a square kilometer. For selected sources, this is becoming comparable with and even exceeding the target collection area anticipated with the Cherenkov Telescope Array.

36th International Cosmic Ray Conference -ICRC2019-

July 24th - August 1st, 2019

Madison, WI, U.S.A.

\footnotetext{
* Speaker.

†https://magic.mpp.mpg. de/. For collaboration list see PoS(ICRC2019)1177
} 